\title{
Pretarsal application of botulinum toxin for treatment of blepharospasm
}

\author{
M Aramideh, B W Ongerboer de Visser, J W M Brans, J H T M Koelman, J D Speelman
}

\begin{abstract}
The response to botulinum toxin type $A$ was compared after two injection techniques in 45 patients with blepharospasm. Initially, patients were treated according to a triple injection technique; two injections into the upper eyelid and one injection into the lower eyelid. Subsequently, without altering the dose, the same patient group received two further injections into the pretarsal portion of the orbicularis oculi muscle of the upper lid. Triple injections were given in 227 treatments, of which $81 \%$ were successful. Mean duration of benefit was $8 \cdot 5$ weeks. Additional pretarsal injections were given in 183 treatment sessions. The number of successful treatments significantly increased, to $95 \%$ ( $P$ $<0.001$ ), and the mean duration of benefit increased to 12.5 weeks $(P<0.001)$. Ptosis occurred significantly less often after pretarsal injections $(P<0.01)$. Patients with combined blepharospasm and involuntary levator palpebrae inhibition responded better to the pretarsal injection technique.
\end{abstract}

$(\Im$ Neurol Neurosurg Psychiatry 1995;59:309-311)
Keywords: dystonia; blepharospasm; botulinum toxin, pretarsal orbicularis oculi

Blepharospasm is characterised by intermittent or persistent involuntary eyelid closure due to spasmodic contractions of the orbicularis oculi muscles, ${ }^{1-6}$ in some instances, in combination with involuntary levator palpebrae inhibition.?

Until the introduction of botulinum toxin type A (BTA) in 1983 by Scott et al, ${ }^{8}$ treatment of blepharospasm had generally been unsuccessful. Although the beneficial effects of BTA are self evident, ${ }^{9}$ there are still several unresolved problems, including the optimal injection sites of BTA.

In this study, we compared the treatment outcomes in a group of patients with blepharospasm, who initially received BTA injected into three sites of the orbicularis oculi muscle, and subsequently received two additional injections into the pretarsal portion of this muscle.
Patients and methods

We studied 45 patients with blepharospasm who received BTA (Dysport) according to both injection techniques successively (table). They had at least two treatments with known outcomes and a follow up of three years until the end of 1994. In 29 patients, EMG was performed from the levator palpebrae and orbicularis oculi muscles before or after the BTA treatment, to investigate the origin of eyelid movement disorder and the cause of unsatisfactory responses. The technique and the results have been reported earlier. ${ }^{710}$ The frequency and severity of spasms of the eyelids were both assessed on a five point scale, as two subcomponents of the blepharospasm rating scale reported by Fahn. ${ }^{11}$ Patients were assessed before injection, and at each follow up visit only at the time of re-injection.

The effectiveness of treatment was based on the patient's report. A patient was considered as a poor responder or a non-responder if more than half of the treatments failed to improve the symptoms. The duration of beneficial effect was the period in weeks after the injection during which the patient noted improvement of symptoms. Initially, patients were re-treated after a fixed period (about

General characteristics of the patients treated

\begin{tabular}{lc}
\hline No of patients & 45 \\
F/M & $33 / 12$ \\
Mean age (y) (range) & $69(27-84)$ \\
Mean age at onset (y) (range) & $59(12-76)$ \\
Mean duration of illness (range) & $10(13-24)$ \\
Mean diagnostic delay (range) & $5(1-15)$ \\
& \\
Complains at onset: & $24(53)$ \\
Increased blinking & $10(22)$ \\
Ophthalmological & $7(16)$ \\
Combination 1 and 2 & $4(9)$ \\
Drooping of eyelid & \\
Extent of dystonia: & $29(64)$ \\
Focal & $15(34)$ \\
Segmental & $1(2)$ \\
Generalised & \\
Previous treatment: & $27(60)$ \\
Medication & $4(9)$ \\
Surgery & $1(2)$ \\
Combination & \\
EMG findings: $\dagger$ & $23(79)$ \\
"Pure" blepharospasm & $2(7)$ \\
Pretarsal blepharospasm & $4(14)$ \\
Blepharospasm and levator inhibition & \\
\hline Unless otherwise stated, values are numbers of patients (\%). \\
$\star$ Dry or watery eyes, photophobia, irritation, or pain in the \\
eyes; $\dagger$ electromyographic recording was performed in 29 \\
patients.
\end{tabular}


three months). If the patient returned before the previous injection had worn off, we did not assess the patient and did not inject BTA. Instead, we made a new appointment usually for four to six weeks later. Subsequently, we chose a more "open door" policy-that is, although a fixed appointment for re-injection was made, the patients could put their appointments either to an earlier date if symptoms had returned, or to a later date if the treatment was still effective.

One vial of BTA containing $500 \mathrm{U}$ was diluted with $5 \mathrm{ml}$ of sterile saline. With the EMG as a guide, BTA was injected into the three components of the orbicularis oculinamely, the orbital, preseptal, and pretarsal portions. Initially, patients were treated according to the triple injection techniquetwo injections at the junction of the orbital and preseptal portions, medially and laterally in the upper lid and one injection laterally in the lower lid. A mean dose of 37 (range 8-85) U was injected on each side. Subsequently, the same patient group, without altering the total amount of BTA, received two injections into the pretarsal portion (a total of $10-15 \mathrm{U}$ on each side), laterally and medially in the upper lid, in addition to triple injections. The data were statistically analysed by Wilcoxon matched pairs signed rank sum test.

\section{Results}

At the baseline level, about $95 \%$ of the patients exhibited forceful eyelid closure, which was present more than $50 \%$ of waking time. The mean values of the severity and frequency of spasms of the eyelids were 3.5 and $4 \cdot 7$ respectively.

Triple injections were given in 227 treatments, of which 186 were successful (81\%). The mean duration of benefit was 8.5 weeks; $17 \%$ of the treatments resulted in ptosis and $2 \%$ in blurred vision. Five patients $(11 \%)$ were poor responders or non-responders. Four of them had a better response after the pretarsal injections and EMG showed a combination of blepharospasm and involuntary levator inhibition in all. One patient with "pure" blepharospasm remained a nonresponder, even after the pretarsal injections. At the time of re-injection, $50 \%$ of the patients had forceful eyelid closure, and in $65 \%$ the involuntary movements were present more than $50 \%$ of waking time. The mean values of the severity and frequency of the spasms, 2.4 and 3.4 respectively, still differed significantly from those at the baseline level $(P<0.001)$.

Additional pretarsal injections were given in 183 treatments. The number of successful treatments significantly increased, to 175 $(95 \%, \mathrm{P}<0.001)$. The mean duration of benefit also significantly increased, to $12 \cdot 6$ weeks $(P<0.001)$. After the additional pretarsal injections, the duration of the positive response increased, particularly in those patients with pure blepharospasm (23 patients with known EMG data) and pretarsal blepharo- spasm (two patients). Additional pretarsal injections resulted in ptosis in $10 \%$ of the treatments, which was significantly less often than in treatments with triple injections $(P<$ $0.001)$. On the other hand, blurred vision occurred in $10 \%$ of the treatments and had a mean duration of one week. After the pretarsal injections and at the time of re-injection, only $10 \%$ of the patients exhibited forceful eyelid closure, and in $39 \%$ the involuntary movements were present more than $50 \%$ of waking time. The mean values of the severity and frequency of the spasms, 1.7 and 3 respectively, still differed significantly from those at the baseline $(P<0.001)$, and from the mean value of severity of spasms after the triple injection $(P<0.01)$.

\section{Discussion}

The present study in 45 patients with blepharospasm showed that BTA application into the pretarsal portion of orbicularis oculi, in addition to triple injections into the preseptal and orbital portions, improves the beneficial response to BTA treatment. After pretarsal injections, the number of successful treatments increased by $14 \%$ and the mean duration of benefit by four weeks. The number of poor responders and non-responders decreased by $9 \%$ and, even at the time of reinjection, the spasms in the eyelids were still significantly less severe. Most of the poor responders and non-responders had a combination of orbicularis oculi spasms and involuntary levator palpebrae inhibition, and responded better to the additional pretarsal injections. The duration of benefit increased particularly in patients with "pure" blepharospasm. Only one patient with pure blepharospasm remained a non-responder after pretarsal injections.

The dramatic improvement of the response to pretarsal BTA application cannot be explained by the fact that the patients were already receiving treatment with BTA before they received additional pretarsal injections, because previous work has shown no increase in efficacy of BTA after repeated injections. ${ }^{1213}$ There may, however, be some overestimation of the positive response to pretarsal injections because dropout patients, who did not respond to the triple injections and did not receive additional pretarsal injections, were not included. It is possible that these patients would not have responded to additional pretarsal injections either. Our unpublished data indicate that the overall successful treatment rate with additional pretarsal injections in the unselected total population of blepharospasm patients is about $85 \%$ to $90 \%$ of the total treatments.

The present study indicates that the activity of the pretarsal portion is probably more often disturbed in blepharospasm than has been assumed, as additional pretarsal injections of BTA increased the magnitude of favourable responses to the treatment in most of the patients. We assumed earlier ${ }^{14}$ that the pretarsal motor units are probably 
more susceptible to alterations in spontaneous supranuclear signals and are less under voluntary control, which may explain the cause of isolated dystonic spasms of the pretarsal portions. ${ }^{15}$

After intramuscular injection, BTA may diffuse to the neighbouring muscles, or may even cause subclinical changes of neuromuscular transmission in distant muscles. ${ }^{16}$ Our results indicate that after preseptal or orbital injection, the diffusion of BTA to the pretarsal portion of the orbicularis is somehow insufficient to induce an optimal paresis, and that direct pretarsal injection of BTA is required. Presumably a higher dose of BTA would eventually cause sufficient paresis of the pretarsal portion through diffusion, but this may considerably increase the possibility of inducing ptosis or diplopia.

Ptosis, as the most frequent and troublesome side effect of BTA, occurred in $17 \%$ of the treatments with triple injections, whereas after pretarsal injections $10 \%$ of the treatments resulted in ptosis. There may be two explanations for this. Firstly, to give two additional pretarsal injections with the same total amount of BTA, we had to give a lower dose of BTA into the preseptal and orbital sites. This may have reduced the chance of BTA diffusion to the levator palpebrae muscle. Secondly, the levator palpebrae becomes aponeurotic already at the level of the superior transverse ligament, some 14 to $20 \mathrm{~mm}$ above the tarsus. ${ }^{17}$ Therefore, pretarsal injections are probably less likely to induce ptosis because of a greater distance between the injection site and neuromuscular junctions of levator palpebrae. By contrast, blurred vision occurred significantly more often after pretarsal injections. According to the patients' reports, this side effect was very mild and could occur with a frequency of 10 to 15 times per day during a few minutes each time. Patients were able to suppress the blurred vision by closing the eyelids gently, sometimes even manually, and then rubbing the eyelids against the eyes. This side effect may have been caused by some disattachment between the eyelids and the surface of the eyes, probably due to paresis of subpretarsal muscle, also known as pars marginalis or pars ciliaris, or the ciliary bundle of Riolan, ${ }^{18}$ which may allow the build up of debris on the corneal surface resulting in refractive distortion.

The mean dose of BTA given in our patients was considerably lower than that often reported by others or than that recommended by the manufacturer. Yet the results are similar. To our knowledge, no study has been performed to find out the optimal dose for inducing sufficient eyelid paresis with the least side effects.

With respect to the increased duration of beneficial effect by additional pretarsal BTA application, it is known that the pretarsal portion is particularly involved in spontaneous, voluntary, and reflex blinking. ${ }^{19}$ Probably our lower dose application into the preseptal or orbital portion and, hence, insufficient pretarsal paresis, allows the earlier recurrence of spontaneous blinks. In fact, the increased blinking frequency is for many patients with blepharospasm the initial sign of loss of effect of BTA and the cause of early complaints. Furthermore, the pretarsal portion is mainly composed of type I muscle fibres. Experimental work on rat calf muscles ${ }^{20}$ showed that the recovery of type I muscle fibres from botulinum toxin is much faster than that of type II fibres. Therefore, it is possible that direct injection of BTA into the pretarsal portion would block more neuromuscular junctions and, hence, would prolong the period of effective paresis.

In conclusion, we suggest that additional injections of BTA into the pretarsal portion of orbicularis oculi muscles on the upper eyelid increase the magnitude of the response to BTA treatment and decrease the number of unsuccessful treatments and ptosis.

We thank Professor J Stam for his helpful comments in the preparation of this manuscript.

1 Marsden CD. Blepharospasm-oromandibular dystonia syndrome (Brueghel's syndrome): a variant of adultonset torsion dystonia? 7 Neurol Neurosurg Psychiatry 1976;39:1204-9.

2 Jankovic J, Ford J. Blepharospasm and orofacial-cervical dystonia: clinical and pharmacological findings in 100 patients. Ann Neurol 1983;13:402-11.

3 Grandas F, Elston J, Quinn N, Marsden CD. Blepharospasm: a review of 264 patients. $\mathcal{f}$ Neurol Neurosurg spasm: a review of 264 pat

4 Elston JS, Marsden CD, Grandas F, Quinn NP. The significance of ophthalmological symptoms in idiopathic blepharospasm. Eye 1988;2:435-9.

5 Berardelli A, Rothwell JC, Day BL, Marsden CD. Pathophysiology of blepharospasm and oromandibular dystonia. Brain 1985;108:593-608.

6 Tolosa E, Marti MJ. Blepharospasm-oromandibular dystonia syndrome (Meige's syndrome): clinical aspects. In: Jankovic J, Tolosa E, eds. Advances in neurology. New York, Raven Press, 1988:73-84.

7 Aramideh M, Ongerboer de Visser BW, Devriese PP, Bour LJ, Speelman JD. Electromyographic features of levator palpebrae superioris and orbicularis oculi muscles in blepharospasm. Brain 1994;117:27-38.

8 Scott AB, Kennedy RA, Stubbs HA. Botulinum A toxin injection as a treatment for blepharospasm. Arch injection as a treatment for

9 Elston JS. Botulinum toxin for blepharospasm. In: Jankovic J, Hallett $M$, eds. Therapy with botulinum toxin. New York, Marcel Dekker, 1994:191-7.

10 Aramideh M, Ongerboer de Visser BW, Koelman JHTM, Bour LJ, Devriese PP, Speelman JD. Clinical and electromyographic features of levator palpebrae superioris muscle dysfunction in involuntary eyelid closure. Mov Disord 1994;9:395-402.

11 Fahn S. Rating scales for blepharospasm. Adv Ophthalmic Plast Reconstr Surg 1985;4:97-101.

12 Dutton JJ, Buckley EG. Botulinum toxin in the management of blepharospasm. Arch Neurol 1986;43:380-2.

13 Biglan AW, May M, Bowers RA. Management of facial spasm with clostridium botulinum toxin, type A spasm with clostridium botulinum toxin, type A (Oculinum) Arch

14 Aramideh M, Ongerboer de Visser BW, Koelman JHTM, Speelman JD. Motor persistence of orbicularis oculi muscle in eyelid opening disorders. Neurology 1995;45: 897-902.

15 Elston JS. A new variant of blepharospasm. $f$ Neurol Neurosurg Psychiatry 1992;55:369-71.

16 Lange DJ, Brin MF, Warner CL, Fahn S, Lovelace RE. Distant effects of local injections of botulinum toxin Muscle Nerve 1987;10:552-5.

17 Anderson RL, Beard C. The levator aponeurosis. Attachments and their clinical significance. Arch Ophthalmol 1977;95:1437-41.

18 Whitnall SE. Anatomy of the human orbit and accessory organs of vision. The eyelids: Oxford: Humphrey Milford, 1932:109-252.

19 Gordon G. Observation upon the movement of the eyelids. Br 7 Ophthalmol 1951;35:339-51.

20 Hassan SM, Jennekens FGI, Wieneke G, Veldman $H$ Elimination of superfluous neuromuscular junctions in the rat calf muscles recovering from botulinum toxininduced paralysis. Muscle Nerve 1994;17:623-31. 